\title{
SKA1 regulates the metastasis and cisplatin resistance of non-small cell lung cancer
}

\author{
LIHUA SHEN $^{1,2^{*}}$, MIN YANG $^{3 *}$, QIONGHUA LIN $^{1}$, ZHONGWEI ZHANG $^{1,2}$, \\ CHANGHONG MIAO $^{1,2}$ and BIAO ZHU ${ }^{1,2}$
}

\author{
${ }^{1}$ Department of Anaesthesia, Critical Care and Pain Medicine, Fudan University Shanghai Cancer Center, Shanghai 200032; \\ ${ }^{2}$ Department of Oncology, Shanghai Medical College, Fudan University, Shanghai 200032; \\ ${ }^{3}$ Department of Respiratory Diseases, Tianjin First Center Hospital, Tianjin 300192, P.R. China
}

Received November 12, 2015; Accepted January 27, 2016

DOI: $10.3892 /$ or.2016.4670

\begin{abstract}
Currently, chemotherapy with platinum-based drugs including cisplatin is the most effective therapy for the treatment of non-small cell lung carcinoma (NSCLC). However, the efficacy of chemotherapy is limited due to commonly developed drug resistance. Spindle and kinetochore-associated complex subunit 1 (SKA1) is part of a complex essential for stabilizing the attachment of spindle microtubules to kinetochores and for maintaining the metaphase plate during mitosis. In the present study, we aimed to investigate the role of SKA1 in the process of metastasis and drug resistance of NSCLC. We completed a series of experiments to investigate the function of SKA1 in NSCLC metastasis and drug resistance including qRT-PCR, immunohistochemistry and western blotting, as well as MTT, BrdU, wounded healing, Transwell and gelatin zymography assays. We demonstrated that the expression levels of SKA1 were elevated in NSCLC and were correlated with cancer progression and malignancy. We also reported that SKA1 positively regulated the proliferation and metastatic ability of NSCLC cells. In addition, we determined that SKA1 contributed to cisplatin resistance in NSCLC cells by protecting these cells from cisplatin-induced cell apoptosis. SKA1 also appeared to regulate the ERK1/2 and the Akt-mediated signaling pathways in NSCLC cells. SKA1 is required for metastasis and cisplatin resistance of non-small cell lung cancer.
\end{abstract}

Correspondence to: Dr Biao Zhu or Dr Changhong Miao, Department of Anaesthesia, Critical Care and Pain Medicine, Fudan University Shanghai Cancer Center, Shanghai 200032, P.R. China E-mail: biaozhufudan@163.com

E-mail:miaochh@aliyun.com

${ }^{*}$ Contributed equally

Key words: SKA1, NSCLC, metastasis, cisplatin, drug resistance

\section{Introduction}

Lung cancer is the most common cause of cancer-related death in both men and women (1), with $\sim 1.5$ million new cases diagnosed annually worldwide. Lung cancer can be histologically divided into two types: small cell lung carcinomas (SCLCs) and non-small cell lung carcinomas (NSCLCs), with the latter accounting for more than $80 \%$ of all lung cancer cases (2). Within NSCLCs, there are also heterogeneous subtypes of tumors, including squamous, adenocarcinoma and large cell carcinomas (1). Once diagnosed, patients with NSCLC are often found to be in advanced stages (3), and are therefore prone to metastasis and recurrence. As a result, despite recent progress, the therapeutic outcome of NSCLC remains poor. Currently, a combination of chemotherapy regimens with platinum-based drugs, particularly cisplatin, is the most effective first-line therapy for the treatment of NSCLC according to clinical guidelines. It has been well established in the literature that the induction of DNA lesions and cell apoptosis is the major mechanism of cisplatin action (4). However, the efficacy of chemotherapy is limited for NSCLC since chemoresistance commonly develops and results in failure of chemotherapy. The development of cisplatin resistance is mediated through multiple mechanisms, including gene mutations or epigenetic changes that influence the uptake and metabolism of drugs by cancer cells $(5,6)$.

Recently, a series of studies suggest the involvement of spindle and kinetochore-associated complex subunit 1 (SKA1) in the growth and proliferation of multiple cancer types, including oral adenosquamous (7) and hepatocellular carcinoma (8), bladder (9), gastric (10), prostate (11) and thyroid cancer (12), and glioblastoma (13). SKA1, along with SKA2 and SKA3, constitute the SKA complex. The SKA complex is localized to the spindle microtubule and on the outer kinetochore interface during mitosis. As it has been shown, this complex is essential for stabilizing the attachment of spindle microtubules to kinetochores and for maintaining the metaphase plate, and is therefore essential for proper chromosome segregation during mitosis (14-16). Efficient depletion of the SKA complex was found to lead to unstable kinetochore microtubule structure formation and severe chromosome 
alignment defects (14). In addition, the SKA complex has been implicated in bypassing of the spindle checkpoint (17) and in the maintenance of sister chromatid cohesion (16).

Although SKA1 has been implicated in the development of multiple cancers, its involvement in the recurrence, metastasis and drug resistance in NSCLC has not been studied. In the present study, we demonstrated that the expression levels of SKA1 were elevated in NSCLC and were correlated with cancer progression and malignancy. We also reported that SKA1 positively regulated the proliferation and metastatic ability of NSCLC cells. Additionally, we determined that SKA1 contributed to cisplatin resistance in NSCLC cells by protecting these cells from cisplatin-induced cell apoptosis. Finally, SKA1 appeared to regulate the ERK1/2 and Akt signaling pathways in NSCLC cells.

\section{Materials and methods}

Reagents and antibodies. TRIzol reagent and Lipofectamine 2000 were purchased from Invitrogen (Carlsbad, CA, USA). The First Strand cDNA Synthesis kit and SYBR Premix Taq were from Takara (Dalian, Liaoning, China). 3-(4,5-Dimethyl2-thiazolyl)-2,5-diphenyl-2H-tetrazolium bromide (MTT), bromodeoxyuridine (BrdU) and the anti-BrdU antibody were purchased from Sigma (St. Louis, MO, USA). DAPI, BCA protein assay and ECL Plus kits were obtained from Beyotime Institute of Biotechnology (Beijing, China). BD BioCoat Matrigel invasion chambers were purchased from BD Biosciences (San Jose, CA, USA).

The primary antibodies against human SKA1 and cleaved caspase-3 were obtained from Abcam (Cambridge, MA, USA). Anti-Bcl-2, anti-Bax, anti-p-ERK1/2, anti-ERK1/2, anti-pAkt, anti-Akt, anti-p21, anti-cyclin D1 and anti-GAPDH were purchased from Santa Cruz Biotechnology (Santa Cruz, CA, USA). The horseradish peroxidase-conjugated anti-rabbit and anti-mouse IgG secondary antibodies were purchased from Santa Cruz Biotechnology. Biotinylated- and Cy3-conjugated anti-rabbit secondary antibodies were purchased form Boster (Wuhan, Hubei, China).

Cell culture and transfection. The human non-small cell lung cancer (NSCLC) cell lines (A549, H23, H520 and H1975) were purchased from the American Type Culture Collection (ATCC; USA) and cultured in RPMI-1640 medium supplemented with $10 \%$ fetal calf serum (FBS), $100 \mathrm{U} / \mathrm{ml}$ penicillin $\mathrm{G}, 100 \mathrm{mg} / \mathrm{ml}$ streptomycin sulfate, and $2 \mathrm{mmol} / \mathrm{l}$ glutamine (all from Gibco, Rockville, MD, USA) at $37^{\circ} \mathrm{C}$ in a humidified incubator under an atmosphere of $5 \% \mathrm{CO}_{2}$ in air.

Human SKA1 cDNA was amplified from A549 cells by PCR and constructed into the pcDNA3 vector (Invitrogen). SKA1 siRNA and control siRNA were purchased from Santa Cruz Biotechnology. Transfections of the vector or siRNA to cells were performed using Lipofectamine 2000 according to the manufacturer's protocol.

Quantitative real-time RT-PCR ( $q R T$-PCR). Total RNA was extracted with TRIzol reagent from NSCLC samples and cell lines according to the manufacturer's instructions. Total RNA $(5 \mu \mathrm{g})$ was reversely transcribed to cDNA using the First Strand cDNA Synthesis kit. The expression of mRNA was examined by qRT-PCR with SYBR Premix Taq and Applied Biosystems 7500 Sequence Detection system. The relative expression levels of mRNA were normalized to GAPDH expression and the amplification results for qRT-PCR were calculated using the $2^{-\Delta \Delta \mathrm{Ct}}$ method. The PCR reaction was performed using $S K A 1$ primers: 5'-TGATGTGCCAGGAAG GTGAC-3' (forward) and 5'-CAAAGGATACAGATGAACA ACAGC-3' (reverse); GAPDH primers: 5'-GTGGACATCCGC AAAGAC-3' (forward) and 5'-AAAGGGTGTAACGCAA CTA-3' (reverse).

Immunohistochemistry. The paraffin-embedded tissue samples from postoperative patients were sectioned into $5-\mu \mathrm{m}$ slices and embedded with paraffin after fixation with $10 \%$ formaldehyde. The samples were then deparaffinized in xylene, rehydrated and the endogenous peroxidase activity was quenched by $3 \%$ hydrogen peroxide. Non-specific binding was blocked with $1 \%$ bovine serum albumin. The sections were then incubated overnight at $4^{\circ} \mathrm{C}$ with the SKA1 primary antibody, and the subsequent secondary antibody followed by the DAB secondary antibody.

MTT and cell number counting assay. MTT assay was used to assess cell proliferation and viability. Briefly, transfected NSCLC cells were seeded at a density of $2.5 \times 10^{3}$ cells/well in a $100 \mu 1$ volume of medium in 96-well plates and allowed to attach overnight. MTT ( $20 \mu \mathrm{l}$ at $5 \mathrm{mg} / \mathrm{ml})$ was added to the medium after treatment. After incubation at $37^{\circ} \mathrm{C}$ for $4 \mathrm{~h}$, the supernatant was aspirated, and $100 \mu \mathrm{l}$ dimethyl sulfoxide (DMSO) was added to each well to dissolved the precipitate. The negative control well contained medium only. Absorbance at $570 \mathrm{~nm}$ was measured by a $96-$ well microplate reader. The survival ratio was calculated using the following equation: Survival ratio $(\%)=\mathrm{OD}_{\text {treated }} / \mathrm{OD}_{\text {control }} \times 100$. For cell counting, NSCLC cells were plated onto $35-\mathrm{mm}$ dishes at $2 \times 10^{3}$ cells/dish. On days $0-6$, the cells were trypsinized and counted with a hemocytometer under a microscope.

$B r d U$ assay. BrdU assay was used to analyze cell proliferation and was carried out according to the manufacturer's recommendations. The NSCLC cells were seeded on a 4-well chamber slide and cultured in growth medium overnight. BrdU-labeled medium was then replaced and incubated at $37^{\circ} \mathrm{C}$ for $3 \mathrm{~h}$. The cell medium was removed and cells were fixed with ethanol for $20 \mathrm{~min}$ at $-20^{\circ} \mathrm{C}$. The cells were permeablized with $0.1 \%$ Triton X-100 in phosphate-buffered saline (PBS) and blocked with 3\% FBS in PBS solution. After washing the cells, antiBrdU reagent was added for $30 \mathrm{~min}$ at $37^{\circ} \mathrm{C}$. The nuclei were counterstained with DAPI. For fluorescence detection, cell images of each treatment were captured with an Olympus inverted microscope.

Wound healing assay. The wound healing assay was used to evaluate the cancer cell motility capacity. Briefly, transfected NSCLC cells were seeded in 6-well plates and cultured overnight. When the culture had reached nearly $90 \%$ confluency, the cell layer was wounded by dragging a 1,000- $\mu 1$ sterile pipette tip through the monolayer and washed with PBS twice to remove cellular debris. Cells were then cultured for up to $48 \mathrm{~h}$ with growing medium. Photographic images of the plates 
were acquired under a microscope and the relative surface traveled by the leading edge was assessed.

Transwell assay. Cell invasion activity was assessed using 24-well BD BioCoat Matrigel invasion chambers ( $8-\mu \mathrm{m}$ pores) according to the manufacturer's instructions. Transfected NSCLC cells $\left(5 \times 10^{4}\right.$ cells $\left./ \mathrm{ml}\right)$ were loaded into the top chamber with serum-free media. In the lower chamber, $450 \mu$ l growing medium with $10 \%$ FBS was added. After $24 \mathrm{~h}$ of cultivation, the medium was removed and the Transwell chamber was gently wiped with a cotton swab. The migrated cells were fixed in $4 \%$ paraformaldehyde, stained with crystal violet solution and counted under a microscope.

Western blotting. NSCLC cells were washed with PBS and incubated in RIPA buffer (150 mM NaCl, $1 \% \mathrm{NP}-40,0.5 \%$ sodium deoxycholate, $0.1 \% \mathrm{SDS}, 50 \mathrm{mM}$ Tris $\mathrm{pH} 8.0,5.0 \mathrm{mM}$ EDTA pH 8.0, $0.5 \mathrm{mM}$ dithiothreitol and $1 \mathrm{mM}$ phenylmethylsulfonyl fluoride) containing protease inhibitor on ice for $30 \mathrm{~min}$, and centrifuged at $12,000 \mathrm{xg}$ for $15 \mathrm{~min}$ at $4^{\circ} \mathrm{C}$. Protein concentration was determined with the BCA protein assay kit and equal amounts of total protein were separated by $10 \%$ SDS page, and the protein was transferred from the gel to the nitrocellulose membrane. The nitrocellulose membrane was then blocked with 5\% BSA for $1 \mathrm{~h}$. The membrane was then probed with a specific primary antibody and gently shaken at $4^{\circ} \mathrm{C}$ overnight. The next day, the membrane was washed three times with TBST buffer, and incubated with secondary antibodies for $1 \mathrm{~h}$ at room temperature. The image was acquired using the ECL plus kit.

Gelatin zymography. Gelatinolytic activity of MMP-2 (gelatinase A) and MMP-9 (gelatinase B) was analyzed by gelatin gel zymography. NSCLC cells were harvested using lysis buffer [0.5 M Tris- $\mathrm{HCl}, \mathrm{pH} 6.8,4 \%$ sodium dodecyl sulfate (SDS), $0.005 \%$ bromophenol blue, $20 \%$ glycerol] at room temperature for $10 \mathrm{~min}$, and an equal amount of total protein was separated on $10 \%$ SDS-polyacrylamide electrophoresis gels containing $0.1 \%$ gelatin. The gels were denatured in denaturing buffer (2.5\% Triton $\mathrm{X}-100)$ for $30 \mathrm{~min}$ at room temperature, and then incubated at $37^{\circ} \mathrm{C}$ overnight in developing buffer (50 mM Tris, $0.2 \mathrm{M} \mathrm{NaCl}, 5 \mathrm{mM} \mathrm{CaCl}_{2}, 0.02 \%$ Brij-35, pH 7.6) allowing the reactivated enzyme to degrade the copolymerized substrate. After incubation, the gels were stained with $0.25 \%$ Coomassie brilliant blue R-250 for $4 \mathrm{~h}$ at room temperature and destained in distilled water containing $30 \%$ methanol and $10 \%$ glacial acetic acid. The areas where the gelatin substrate had been degraded by gelatinases developed into white lines on a dark background.

Patient samples. A total of 65 samples were surgically obtained from NSCLC patients at the Department of Thoracic Surgery, Fudan University Shanghai Cancer Center. The clinical characteristics of all of the patients were recorded (Table I). The stage of NSCLC was categorized according to surgical and pathological findings, which were based on the guidelines described by the 6 th edition of AJCC/UICC. Written informed consent was obtained from all patients. The present study was approved by the Institutional Ethical Review Boards of Fudan University Shanghai Cancer Center.
Table I. Correlation between SKA1 and the clinicopathological characteristics of the 65 NSCLC patients.

\begin{tabular}{|c|c|c|c|c|}
\hline \multirow[b]{2}{*}{ Features } & \multirow[b]{2}{*}{$\begin{array}{l}\text { No. of } \\
\text { pts. }\end{array}$} & \multicolumn{2}{|c|}{ SKA1 } & \multirow[b]{2}{*}{ P-value } \\
\hline & & $\begin{array}{l}\text { Low } \\
\mathrm{n}(\%)\end{array}$ & $\begin{array}{l}\text { High } \\
\mathrm{n}(\%)\end{array}$ & \\
\hline All patients & 65 & $32(49.2)$ & $33(50.8)$ & \\
\hline Age (years) & & & & 0.108 \\
\hline$<60$ & 35 & $14(40.0)$ & $21(60.0)$ & \\
\hline$\geq 60$ & 30 & $18(60.0)$ & $12(40.0)$ & \\
\hline Gender & & & & 0.302 \\
\hline Male & 47 & $25(53.2)$ & $22(46.8)$ & \\
\hline Female & 18 & $7(38.9)$ & $11(61.1)$ & \\
\hline Smoking status & & & & 0.362 \\
\hline Smokers & 26 & $11(42.3)$ & $15(57.7)$ & \\
\hline Non-smokers & 39 & $21(53.8)$ & $18(46.2)$ & \\
\hline Histology & & & & $0.034^{\mathrm{a}}$ \\
\hline $\begin{array}{l}\text { Squamous cell } \\
\text { carcinoma }\end{array}$ & 21 & $9(42.9)$ & $12(57.1)$ & \\
\hline Adenocarcinoma & 32 & $18(56.3)$ & $14(43.7)$ & \\
\hline Others $^{b}$ & 12 & $5(41.7)$ & $7(58.3)$ & \\
\hline Tumor size $(\mathrm{cm})$ & & & & 0.031 \\
\hline$<3$ & 24 & $16(66.7)$ & $8(33.3)$ & \\
\hline$\geq 3$ & 41 & $16(39.0)$ & $25(61)$ & \\
\hline TNM stage & & & & 0.054 \\
\hline I-II & 39 & $23(59.0)$ & $16(41.0)$ & \\
\hline III-IV & 26 & 9 (34.6) & $17(65.4)$ & \\
\hline Lymph node metastasis & & & & 0.018 \\
\hline Metastasis & 36 & $13(36.1)$ & $23(63.9)$ & \\
\hline Non-metastasis & 29 & $19(65.5)$ & $10(34.5)$ & \\
\hline Differentiation & & & & 0.009 \\
\hline Well and moderate & 28 & $19(67.9)$ & $9(32.1)$ & \\
\hline Poor & 37 & $13(35.1)$ & $24(64.9)$ & \\
\hline
\end{tabular}

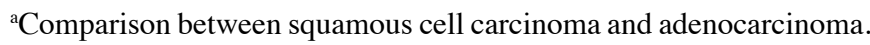
${ }^{\mathrm{b}}$ Other NSCLC include adenosquamous, large-cell and mucoepidermoid carcinoma and carcinosarcoma. NSCLC, non-small cell lung carcinoma; pts., patients.

Statistical analysis. Data were analyzed using SPSS software 19.0 for Windows (SPSS, Inc., Chicago, IL, USA). Results represent the mean values of at least three different experiments and are expressed as the mean \pm SD and were analyzed by t-test or one-way ANOVA. $\mathrm{P}<0.05$ was considered to indicate a statistically significant result.

\section{Results}

SKA1 expression is upregulated in NSCLC. It has been reported that SKA1 may play a role in a series of human cancers. However, its function in NSCLC is unknown. In order to investigate the involvement of SKA1 in the development of NSCLC, we first examined the levels of SKA1 expression in cancer tissues 
A

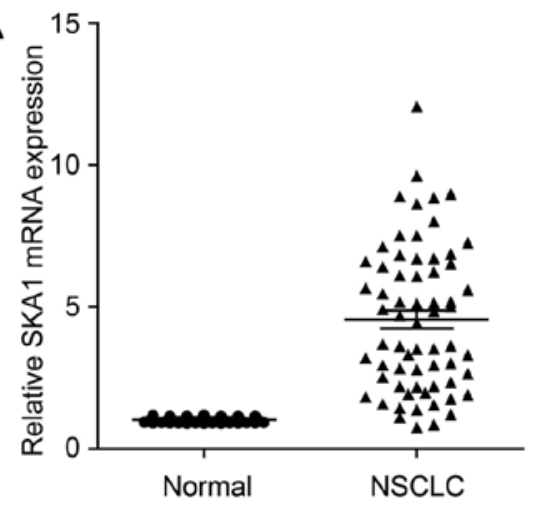

C

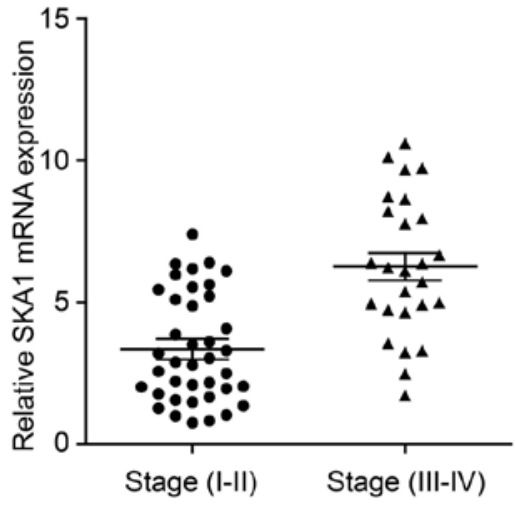

B

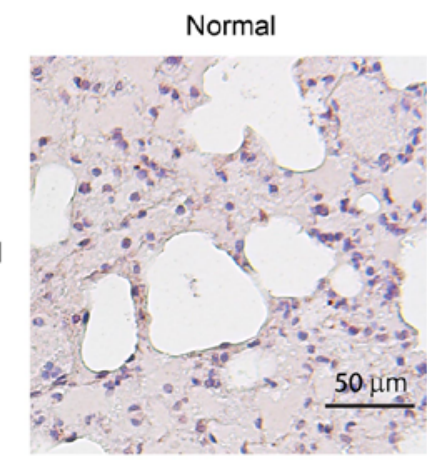

D

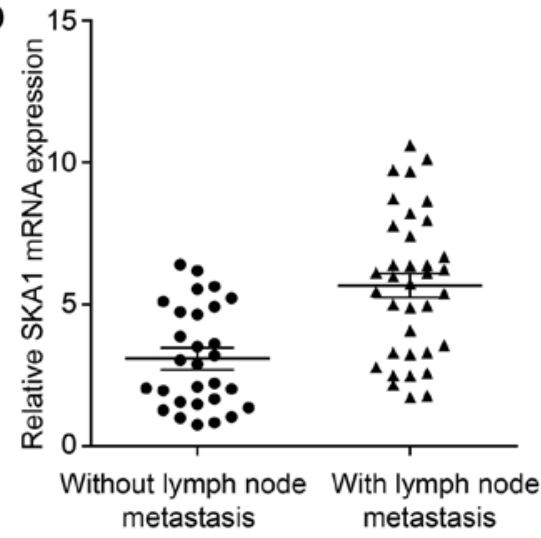

NSCLC

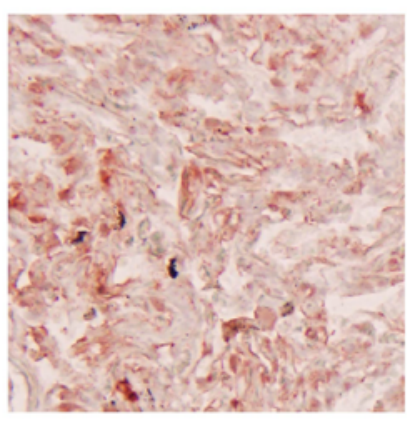

E

GAPDH

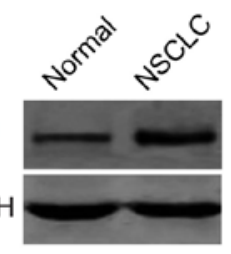

Figure 1. SKA1 expression is upregulated in NSCLC. (A) Relative expression levels of SKA1 in NSCLC and adjacent normal tissues as determined by qRT-PCR analysis (P<0.001). (B) Immunohistochemical staining of SKA1 in NSCLC and adjacent normal tissues. (C) Relative expression levels of SKA1 in tissue samples from NSCLC stages I-IV as determined by qRT-PCR analysis ( $<<0.001)$. (D) Relative expression levels of SKA1 in NSCLC samples with and without lymph node metastasis as determined by qRT-PCR analysis $(\mathrm{P}<0.001)$. (E) SKA1 expression in NSCLC patient tissue analyzed by western blotting.

from 64 NSCLC patients. We found that the expression of SKA1 was significantly elevated in NSCLC tissues, compared to that in the adjacent normal lung tissues (Fig. 1A). This result was also confirmed by immunohistochemical staining of the NSCLC samples for SKA1 (Fig. 1B). We next sought to determine whether the expression levels of SKA1 were associated with the pathological progression of NSCLC. We found that the levels of SKA1 were significantly increased in cancer samples from stage III-IV patients, than the levels in samples from stage I-II patients (Fig. 1C; Table I). Furthermore, we examined the correlation between SKA1 expression levels and cancer metastasis of NSCLC. The expression levels of SKA1 in 36 NSCLC samples with lymph node metastasis were significantly higher than those in 29 samples without lymph node metastasis (Fig. 1D; Table I). Elevated SKA1 expression was further confirmed by western blotting using NSCLC patient tissue (Fig. 1E). Taken together, these results revealed that the expression levels of SKA1 were upregulated in NSCLC and were correlated with cancer progression and malignancy.

$S K A 1$ regulates the proliferation, migration and invasion of NSCLC cells. In order to investigate the functional roles of SKA1 in an in vitro setting, we first examined the levels of SKA1 expression in four NSCLC cell lines (A549, H23, H520 and H1975), and determined that the levels of endogenous SKA1 expression were highest in H520 cells and were lowest in A549 cells (Fig. 2A). Therefore, in order to obtain most pronounced changes in SKA1 expression, these two cell lines were respectively selected to perform in vitro loss- and gain-of-function experiments. We effectively reduced SKA1 expression in the $\mathrm{H} 520$ cells by siRNA transfection, and increased SKA1 expression in the A549 cells by transfection of a plasmid overexpressing SKA1 (Fig. 2B). We then carried out MTT, cell counting and BrdU assays to examine cell proliferation in the H520 and A549 cells with modified SKA1 expression. We found that the knockdown of SKA1 expression in the H520 cells significantly reduced cell proliferation, as evidenced by cellular metabolic activities (Fig. 2C), cell numbers (Fig. 2D) and percentage of cells in active division (Fig. 2E). In contrast, overexpression of SKA1 in the A549 cells significantly increased cell proliferation (Fig. 2C-E). Furthermore, we also examined the migration and invasion activities of the H520 and A549 cells with modified SKA1 expression using both wound healing and Transwell invasion assays. We found that reduced expression of SKA1 in the H520 cells led to significantly decreased ability of both cell migration (Fig. 3A) and cell invasion (Fig. 3B), while elevated expression of SKA1 in the A549 cells resulted in significantly increased ability of cell migration (Fig. 3A) and invasion (Fig. 3B). Collectively, these results indicated that SKA1 positively regulated the proliferation and metastatic ability of the NSCLC cells.

SKA1 mediates NSCLC cell sensitivity to cisplatin. Since SKA1 plays roles in the regulation of NSCLC cell proliferation and 
A

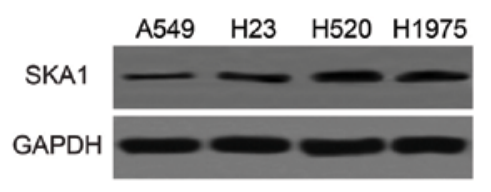

C
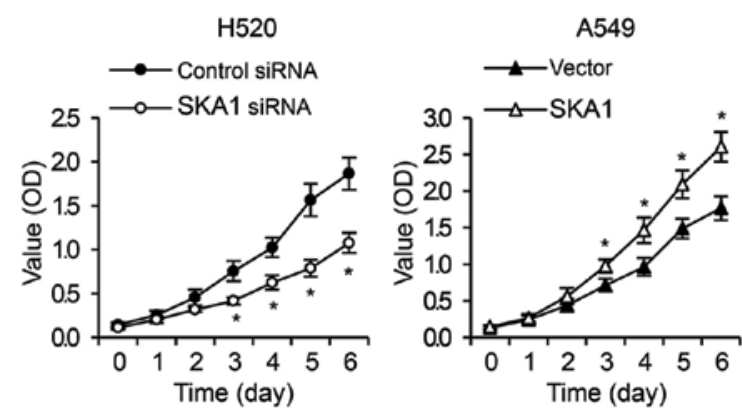

E

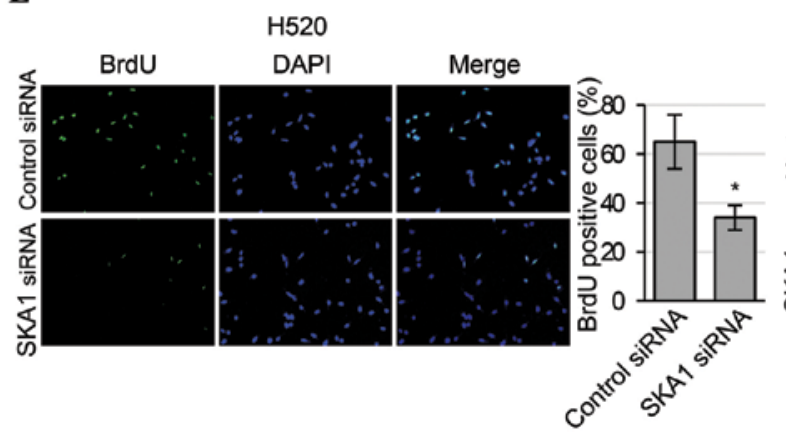

B

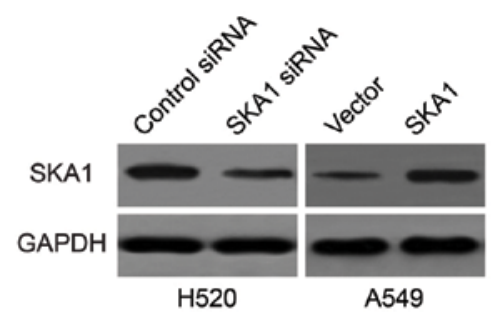

D
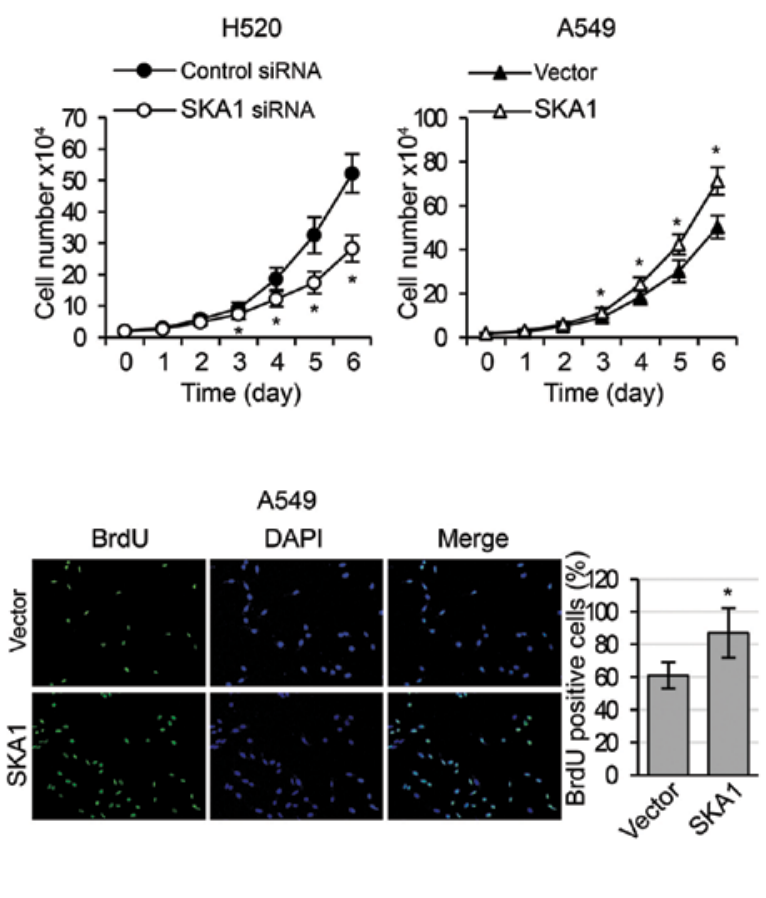

Figure 2. SKA1 positively regulates the proliferation of NSCLC cells. (A) Protein levels of SKA1 in four NSCLC cell lines (A549, H23, H520 and H1975) as determined by western blot analysis. (B) Protein levels of SKA1 in H520 cells transfected with siRNA control or siRNA against SKA1, and in A549 cells transfected with an empty plasmid or a plasmid overexpressing SKA1, as determined by western blot analysis. (C) MTT, (D) cell counting and (E) BrdU assays in H520 cells transfected with siRNA control or siRNA against SKA1, and in A549 cells transfected with an empty plasmid or a plasmid overexpressing SKA1. ${ }^{*} \mathrm{P}<0.05$ compared with the control cells.

metastasis, we next sought to determine whether SKA1 expression levels were associated with the sensitivity of NSCLC cells to cisplatin treatment. We first found that cisplatin treatment substantially increased the levels of SKA1 mRNA (Fig. 4A) and protein (Fig. 4B) in both the H520 and A549 cells. In order to assess the function of SKA1 in regulating NSCLC cell sensitivity to cisplatin, we performed MTT cell viability assay in the H520 and A549 cells with modified SKA1 expression following treatment with different concentrations $(0,2$, $4,8,16,32$ or $64 \mu \mathrm{mol} / \mathrm{l}$ ) of cisplatin for $48 \mathrm{~h}$. We found that while increasing concentrations of cisplatin led to decreased cell viability in both the H520 and A549 cells, knockdown of SKA1 in the H520 cells further reduced cell viability, and overexpression of SKA1 significantly enhanced cell viability in the A549 cells treated with cisplatin (Fig. 5A). In addition, we also analyzed the time course of NSCLC cell viability following treatment with $8 \mu \mathrm{mol} / 1$ cisplatin. Consistent with previous findings, we found that downregulation of SKA1 by siRNA in the H520 cells led to significantly decreased levels of NSCLC cell viability, and that SKA1 overexpression in the A549 cells resulted in significantly higher cell viability in the NSCLC cell lines (Fig. 5B). Furthermore, we examined the levels of several key proteins involved in the apoptosis signaling pathway in NSCLC cells with modified expression levels of SKA1. We determined by western blot analysis that in the H520 cells with SKA1 knockdown, the levels of pro-apoptotic proteins including cleaved caspase-3 and Bax were significantly higher, and the levels of anti-apoptotic proteins including Bcl-2 were significantly lower. The opposite results were observed in the A549 cells with SKA1 overexpression (Fig. 5C). Taken together, these data support the hypothesis that SKA1 protects NSCLC cells from cisplatin-induced cell apoptosis.

SKA1 regulates the ERK1/2 and Akt signaling pathways in NSCLC cells. It has been reported that SKA1 regulates the activities of ERK1/2 and Akt signaling in other cancer types (9). In order to determine whether SKA1 also modulates these pathways in NSCLC, we examined the levels of phosphorylated ERK1/2 and phosphorylated Akt in the H520 and A549 cells with modified SKA1 expression. We found that the levels of both phosphorylated ERK $1 / 2$ and phosphorylated Akt were significantly lower in the H520 cells with SKA1 
A

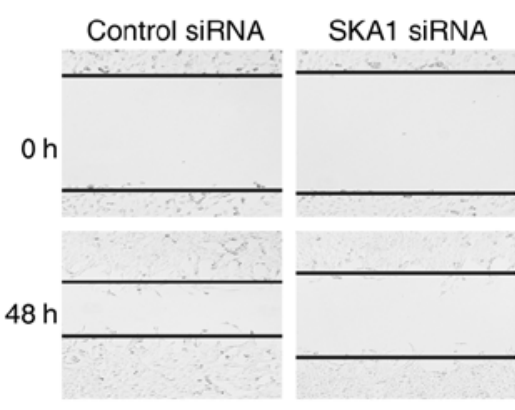

B

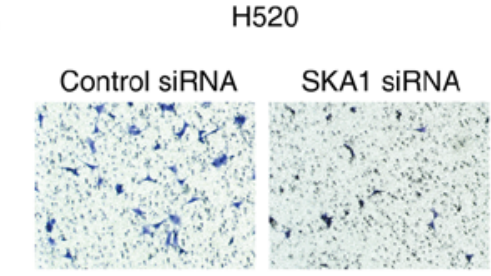

H520
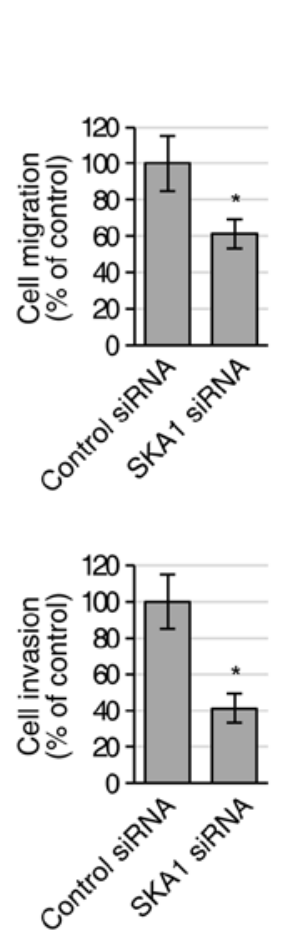

A549

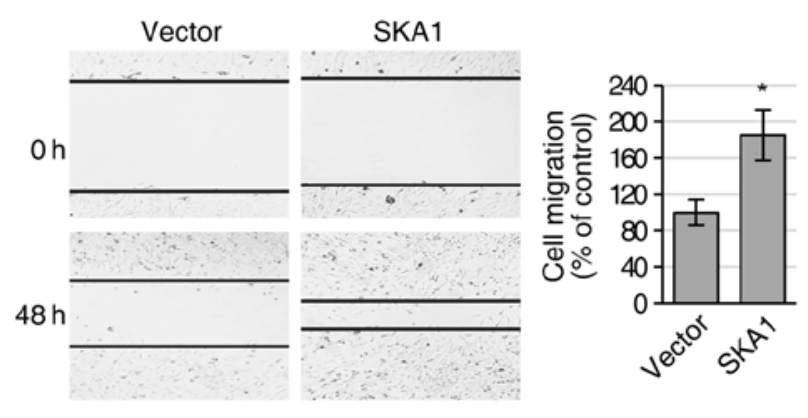

A549
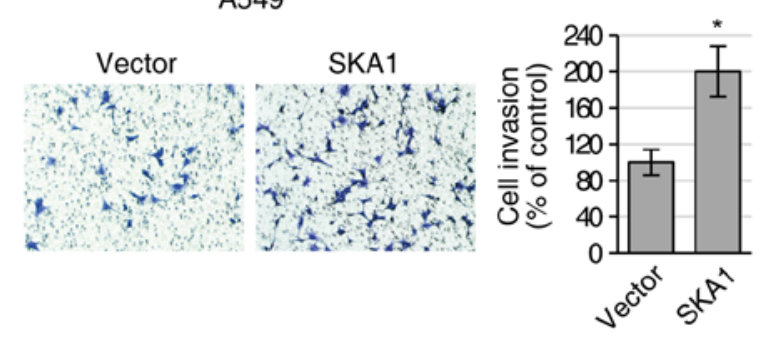

Figure 3. SKA1 positively regulates the migration and invasion of NSCLC cells. (A) Wound healing and (B) Transwell cell invasion assays in H520 cells transfected with siRNA control or siRNA against SKA1, and in A549 cells transfected with an empty plasmid ora plasmid overexpressing SKA1. "P<0.05 compared with the control cells.
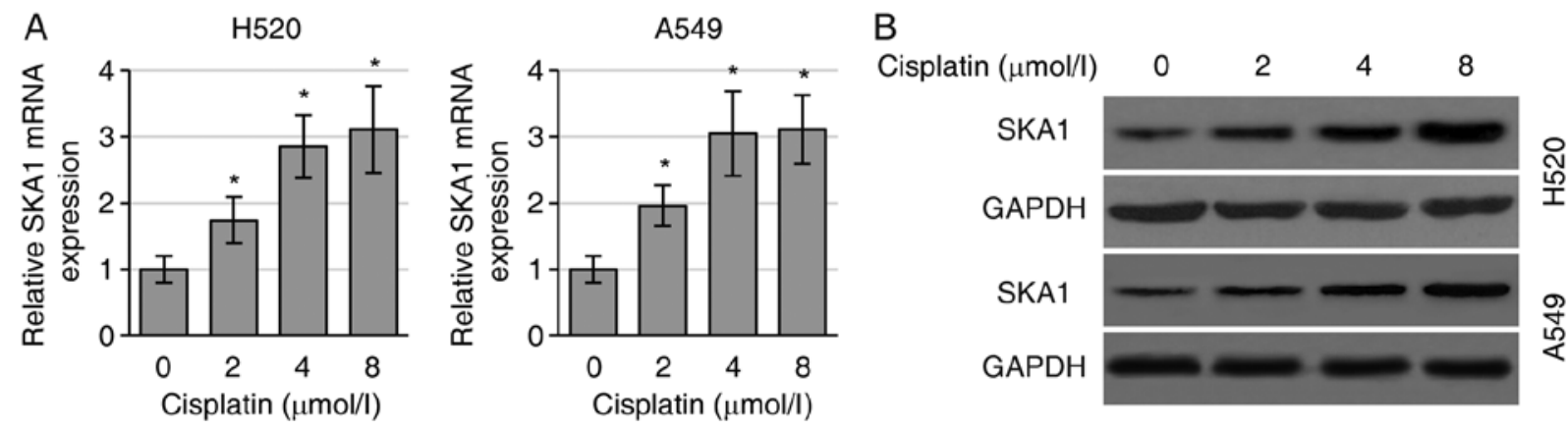

Figure 4. Cisplatin induces SKA1 expression in NSCLC cells. (A) Relative mRNA levels of SKA1 as determined by qRT-PCR analysis and (B) protein levels of SKA1 as determined by western blot analysis in H520 and A549 NSCLC cells treated with the indicated concentrations of cisplatin for $48 \mathrm{~h}$. ${ }^{*} \mathrm{P}<0.05 \mathrm{compared}$ to no cisplatin treatment $(0 \mu \mathrm{mol} / \mathrm{l})$.

knockdown, and were significantly higher in the A549 cells with SKA1 overexpression (Fig. 6A). We next examined the levels of downstream target proteins of the ERK1/2 and the Akt signaling pathways. Consistent with the regulation patterns of these pathways, we found that the levels of p21 were elevated, and the levels of cyclin D1 were reduced with SKA1 knockdown, and that the levels of p21 were reduced, and the levels of cyclin D1 were elevated with SKA1 overexpression (Fig. 6B). In addition, as effectors of the ERK1/2 and the Akt signaling pathways, we also found that the levels of MMP-2 and MMP-9 were decreased by the knockdown of SKA1, and increased by the overexpression of SKA1 (Fig. 6C) as demonstrated in the gelatin zymography assay. In summary, these data indicated that the activities of ERK1/2 and Akt signaling were regulated by SKA1 in the NSCLC cells.

\section{Discussion}

In the present study, we investigated the functional association between SKA1 and the development of NSCLC. We demonstrated that the expression levels of SKA1 were elevated in NSCLC and were correlated with cancer progression and metastasis. We also found that SKA1 positively regulated the proliferation, migration and invasion of NSCLC cells. Importantly, we found that SKA1 contributed to cisplatin resistance in the NSCLC cells by protecting these cells from apoptosis induced by cisplatin. The ERK1/2 and Akt signaling pathways, which play essential roles in cancer development, appeared to be regulated by SKA1 in the NSCLC cells.

Elevated SKA1 expression has been shown by a series of recent studies to be associated with aggressive progression 
A

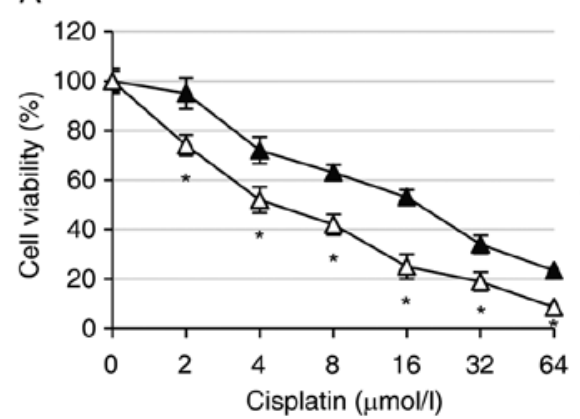

$\mathrm{B}$

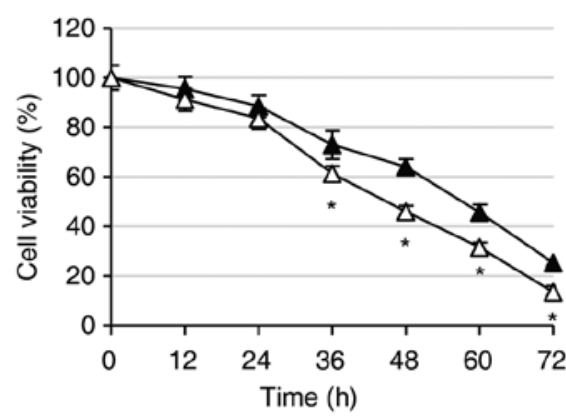

$\longrightarrow$ Control siRNA

$\triangle-$ SKA1 SiRNA

$\longrightarrow$ - Control siRNA
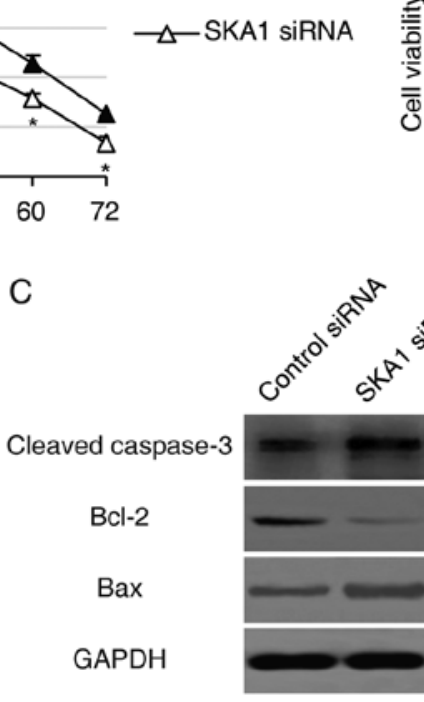

H520

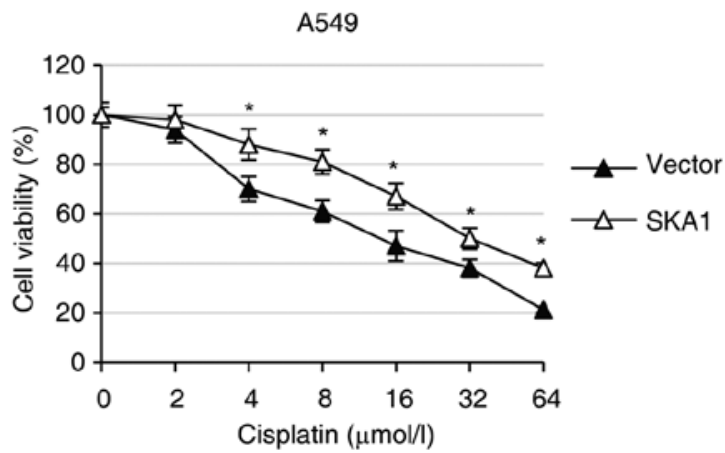

A549

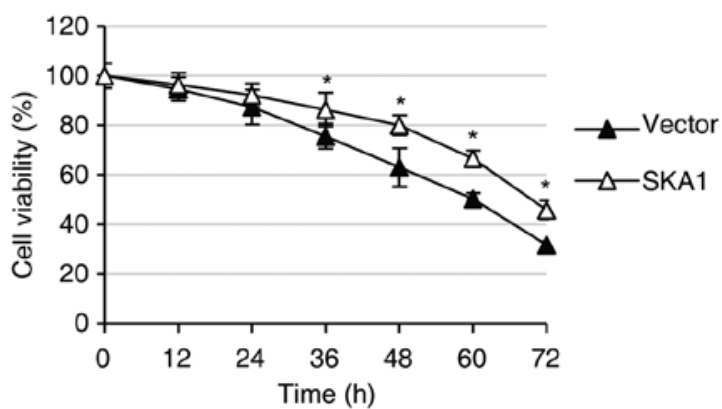

Figure 5. SKA1 protects NSCLC cells from cisplatin-induced cell apoptosis. (A) MTT cell viability assays in H520 cells transfected with siRNA control or siRNA against SKA1, and in A549 cells transfected with an empty plasmid or a plasmid overexpressing SKA1, treated with the indicated concentrations of cisplatin for $48 \mathrm{~h}$. (B) MTT cell viability assays in H520 cells transfected with siRNA control or siRNA against SKA1, and in A549 cells transfected with an empty plasmid or a plasmid overexpressing SKA1, treated with $8 \mu \mathrm{mol} / 1$ cisplatin for the indicated time periods. (C) Protein levels of cleaved caspase-3, Bcl-2 and Bax in H520 cells transfected with siRNA control or siRNA against SKA1, and in A549 cells transfected with an empty plasmid or a plasmid overexpressing SKA1, as determined by western blot analysis. "P<0.05 compared with the control cells.

A

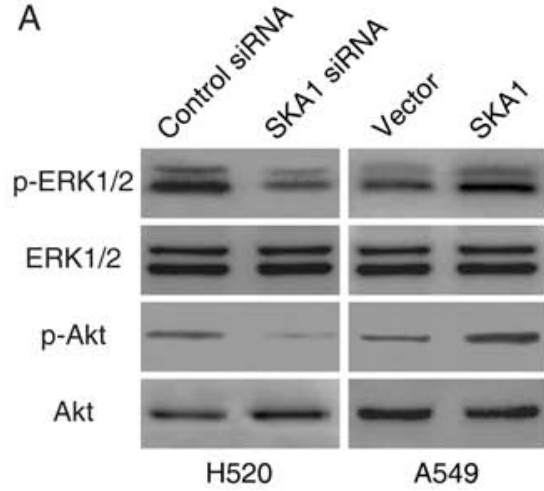

B

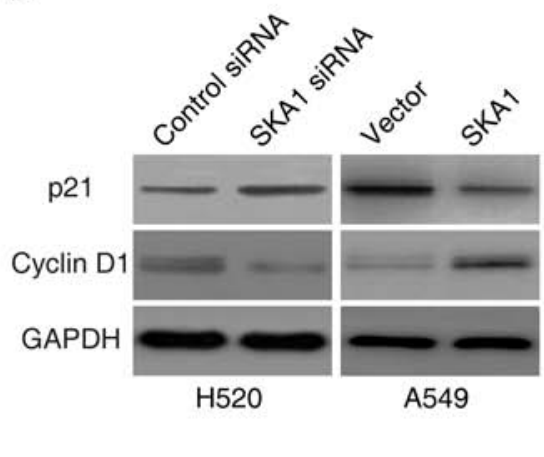

C

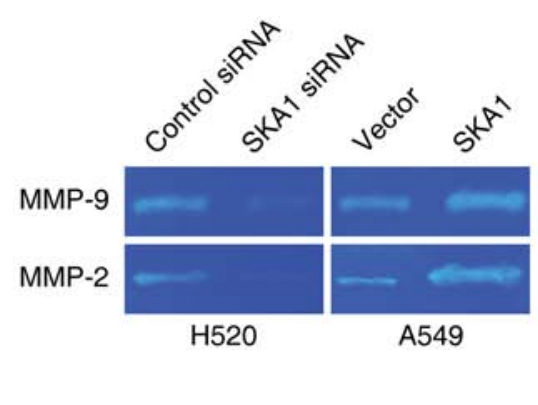

Figure 6. SKA1 regulates the ERK1/2 and Akt signaling pathways in NSCLC cells. (A) Protein levels of p-ERK1/2 and p-Akt in H520 cells transfected with siRNA control or siRNA against SKA1, and in A549 cells transfected with an empty plasmid or a plasmid overexpressing SKA1, as determined by western blot analysis. (B) Protein levels of p21 and cyclin D1 in H520 cells transfected with siRNA control or siRNA against SKA1, and in A549 cells transfected with an empty plasmid or a plasmid overexpressing SKA1, as determined by western blot analysis. (C) Protein levels of MMP-2 and MMP-9 in H520 cells transfected with siRNA control or siRNA against SKA1, and in A549 cells transfected with an empty plasmid or a plasmid overexpressing SKA1, as determined by gelatin zymography assay. 
and poor outcome in several cancer types. In papillary thyroid carcinoma, a significant correlation was noted between SKA1 expression and clinical stage and extrathyroid invasion, and patients with high SKA1 expression were likely to relapse after surgery (12). SKA1 has also been shown to be significantly overexpressed in gastric cancer tissues, and SKA1 silencing significantly inhibited gastric cancer cell colony formation, and led to $S$ phase cell cycle arrest (10). Consistent with these studies, the present study confirmed the relationship between SKA1 expression and the progression of NSCLC. Significantly, for the first time, we established the relationship between SKA1 expression and resistance to cisplatin.

We provided convincing evidence that modulation of SKA1 expression affects cisplatin sensitivity in NSCLC cell lines. It has been reported by previous studies that activation of the ERK1/2 and Akt signaling pathways is associated with cisplatin resistance in lung cancer $(18,19)$. In the present study, we found that the expression levels of SKA1 were positively correlated with the activities of the ERK1/2 and Akt signaling pathways. We noted that the baseline levels of p-ERK1/2 and p-Akt were higher in the H520 cells, which had higher levels of SKA1 expression, compared to those in the A547 cells, which had lower levels of SKA1 expression. Knockdown and overexpression of SKA1 also resulted in consistent changes in the activities of the ERK1/2 and Akt signaling pathways. The ERK1/2 and Akt signaling pathways play essential roles in many aspects of cancer development, including regulation of cell survival and apoptosis, cell proliferation, cell cycle progression and cell matrix interaction $(20,21)$. Therefore, it is reasonable to hypothesize that the mechanism of SKA1 in the regulation of NSCLC progression and drug resistance could be through the modulation of ERK1/2 and Akt signaling. We examined the effects of SKA1 on a series of downstream targets of the ERK1/2 and Akt signaling pathways and confirmed that these targets were also regulated by SKA1. Most studies concerning SKA1 have focused on its functions with kinetochore-microtubule structure during mitosis or meiosis. However, the present study revealed that this protein clearly has other functions.

Notably, in our investigation, we noted that cisplatin treatment led to increased levels of SKA1 in NSCLC cell lines. This result, combined with the findings that SKA1 overexpression protected cells from cisplatin-induced apoptosis, suggest that upregulation of SKA1 may be part of an adaptive response of NSCLC cells to chemotherapy drugs, and may therefore serve as an intrinsic player in the cellular mechanism following chemotherapy treatment. The detailed mechanism of SKA1 upregulation by cisplatin is not currently known, nor do we have evidence that the same response may occur in vivo. Although further investigation is needed to answer these important questions, SKA1 could potentially be a therapeutic target in combating drug resistance in the chemotherapy of NSCLC.

\section{References}

1. Cersosimo RJ: Lung cancer: A review. Am J Health Syst Pharm 59: 611-642, 2002.
2. Parsons A, Daley A, Begh R and Aveyard P: Influence of smoking cessation after diagnosis of early stage lung cancer on prognosis: Systematic review of observational studies with meta-analysis. BMJ 340: b5569, 2010.

3. Sakashita S, Sakashita M and Sound Tsao M: Genes and pathology of non-small cell lung carcinoma. Semin Oncol 41: 28-39, 2014.

4. Galluzzi L, Senovilla L, Vitale I, Michels J, Martins I, Kepp O, Castedo $\mathrm{M}$ and Kroemer G: Molecular mechanisms of cisplatin resistance. Oncogene 31: 1869-1883, 2012.

5. Trédan O, Galmarini CM, Patel K and Tannock IF: Drug resistance and the solid tumor microenvironment. J Natl Cancer Inst 99: 1441-1454, 2007.

6. Dasari S and Tchounwou PB: Cisplatin in cancer therapy: Molecular mechanisms of action. Eur J Pharmacol 740: 364-378, 2014.

7. Zhang B, Li KY, Chen HY, Pan SD, Jiang LC, Wu YP and Liu SW: Spindle and kinetochore associated complex subunit 1 regulates the proliferation of oral adenosquamous carcinoma CAL-27 cells in vitro. Cancer Cell Int 13: 83, 2013.

8. Qin X, Yuan B, Xu X, Huang H and Liu Y: Effects of short interfering RNA-mediated gene silencing of SKA1 on proliferation of hepatocellular carcinoma cells. Scand J Gastroenterol 48: 1324-1332, 2013

9. Tian F, Xing X, Xu F, Cheng W, Zhang Z, Gao J, Ge J and Xie H: Downregulation of $S K A l$ gene expression inhibits cell growth in human bladder cancer. Cancer Biother Radiopharm 30: 271-277, 2015.

10. Sun W, Yao L, Jiang B, Guo L and Wang Q: Spindle and kinetochore-associated protein 1 is overexpressed in gastric cancer and modulates cell growth. Mol Cell Biochem 391: 167-174, 2014.

11. Li J, Xuan JW, Khatamianfar V, Valiyeva F, Moussa M, Sadek A, Yang BB, Dong BJ, Huang YR and Gao WQ: SKA1 over-expression promotes centriole over-duplication, centrosome amplification and prostate tumourigenesis. J Pathol 234: 178-189, 2014.

12. Dong C, Wang XL and Ma BL: Expression of spindle and kinetochore-associated protein 1 is associated with poor prognosis in papillary thyroid carcinoma. Dis Markers 2015: 616541, 2015.

13. Shi X, Chen X, Peng H, Song E, Zhang T, Zhang J, Li J, Swa H, Li Y, Kim S, et al: Lentivirus-mediated silencing of spindle and kinetochore-associated protein 1 inhibits the proliferation and invasion of neuronal glioblastoma cells. Mol Med Rep 11: 3533-3538, 2015.

14. Gaitanos TN, Santamaria A, Jeyaprakash AA, Wang B, Conti E and Nigg EA: Stable kinetochore-microtubule interactions depend on the Ska complex and its new component Ska3/C13Orf3. EMBO J 28: 1442-1452, 2009.

15. Raaijmakers JA, Tanenbaum ME, Maia AF and Medema RH: RAMA1 is a novel kinetochore protein involved in kinetochoremicrotubule attachment. J Cell Sci 122: 2436-2445, 2009.

16. Theis M, Slabicki M, Junqueira M, Paszkowski-Rogacz M, Sontheimer J, Kittler R, Heninger AK, Glatter T, Kruusmaa K, Poser I, et al: Comparative profiling identifies C13orf3 as a component of the Ska complex required for mammalian cell division. EMBO J 28: 1453-1465, 2009.

17. Hanisch A, Silljé HH and Nigg EA: Timely anaphase onset requires a novel spindle and kinetochore complex comprising Ska1 and Ska2. EMBO J 25: 5504-5515, 2006.

18. Wang M, Liu ZM, Li XC, Yao YT and Yin ZX: Activation of ERK1/2 and Akt is associated with cisplatin resistance in human lung cancer cells. J Chemother 25: 162-169, 2013.

19. Chen TJ, Zhou YF, Ning JJ, Yang T, Ren H, Li Y, Zhang S and Chen MW: NBM-T-BMX-OS01, an osthole derivative, sensitizes human lung cancer A549 cells to cisplatin through AMPKdependent inhibition of ERK and Akt Pathway. Cell Physiol Biochem 36: 893-906, 2015.

20. Stinchcombe TE and Johnson GL: MEK inhibition in non-small cell lung cancer. Lung Cancer 86: 121-125, 2014.

21. Yip PY: Phosphatidylinositol 3-kinase-AKT-mammalian target of rapamycin (PI3K-Akt-mTOR) signaling pathway in non-small cell lung cancer. Transl Lung Cancer Res 4: 165-176, 2015. 